\title{
Crowdfunding und Crowdsourcing in der Praxis: Neue Geschäfts- und Rechtsmodelle der Netzliteratur
}

\author{
Thomas Ernst"
}

In der Literaturwissenschaft ist der disruptive Charakter des Schreibens für digitale und soziale Medien sowie für die Nutzung historisch gewachsener Kategorien wie ,Autor ${ }^{6}$, ,Werk ${ }^{6}$ und ,geistiges Eigentum ${ }^{6}$ noch nicht umfassend zur Kenntnis genommen worden. Breit rezipierte literatur- oder medienwissenschaftliche Werke wie jene von Philipp Theisohn oder Monika Dommann verstehen sich entweder als Verteidigungsschriften eines , literarischen Eigentums' oder schrecken noch vor einordnenden Bewertungen der Digitalisierung zurück. ${ }^{2}$ Da das Konstrukt des ,geistigen Eigentums ${ }^{6}$ für das heutige Urheberrecht jedoch fundamental ist und historisch auf Konstruktionen von Autorschaft und Werk-Begriffen im literarischen wie im philosophischen Diskurs Ende des 18. Jahrhunderts zurückgeht, ist es eine Aufgabe der Literaturwissenschaft, den aktuellen medialen Entwicklungen kritisch nachzuspüren.

Dabei ist an anderer Stelle ausführlicher rekonstruiert worden, wie literarische Werk- und Autorschaftsbegriffe sowie die Existenz des Gegenstands, gedrucktes Buch' historisch zur Entwicklung des Konstrukts ,geisti-

* Prof. Dr. Thomas Ernst ist Germanist und Literaturwissenschaftler an der Universiteit Antwerpen sowie an der Universiteit van Amsterdam. Seine Forschungsschwerpunkte sind unter anderem das Geistige Eigentum in der Buch- und der digitalen Kultur sowie subversive, interkulturelle und mehrsprachige Literaturen.

** Ich danke den Teilnehmern des ENVIV-Buchlaunchs am 11. Mai 2013 in der Evangelischen Akademie Tutzing, den Teilnehmern der Konferenz Managing Popular Culture in Karlsruhe vom 1. Februar 2014, Krystian Woznicki und der Berliner Gazette, den Diskutierenden der Tipping Points-Konferenz in Berlin sowie Lioba Foit für wertvolle Hinweise.

1 Kategorien wie ,Autor ${ }^{\varsigma}$, ,Leser', ,Werk' und ,geistiges Eigentum werden in diesem Beitrag regelmäßig durch einfache Anführungszeichen markiert, damit ihr konstruktiver Charakter und ihr historischer Bedeutungswandel deutlicher wird. Ein ähnliches Verfahren findet sich auch in: Woodmansee, On the Author Effect, $15 \mathrm{ff}$.

2 Vgl. Dommann, Geschichte des Copyrights, 296; Theisohn, Literarisches Eigentum, 121-124. 
ges Eigentum' beigetragen haben. ${ }^{3}$ Dieser Beitrag greift dieses literaturwissenschaftliche Wissen kurz auf und verknüpft es mit rechtswissenschaftlichen Perspektiven auf den digitalen Medienwandel. Dabei formuliert er die Notwendigkeit, die Vorstellungen des, geistigen Eigentums' und seine urheberrechtlichen Implikationen in Frage zu stellen und zudem Standards für die kulturelle Produktion in Sozialen Medien zu bestimmen und rechtlich abzusichern (1.).

Anschließend legt er eine Fallstudie aus dem weiten Feld der Netzliteratur vor. Eine neue Version ist verfügbar von Dirk von Gehlen war 2012 das erste größere Buchprojekt, das im deutschsprachigen Raum über die WebPlattform Startnext ein erfolgreiches Crowdfunding durchführte und zugleich Elemente des Crowdsourcing in seinen Produktionsprozess einbeziehen wollte (2.). Die Analyse will untersuchen, inwiefern dieses Projekt tatsächlich als ein Tipping Point für die Entwicklung neuer Geschäfts- und Rechtsmodelle der Literatur zu bewerten und seinen selbst formulierten Ansprüchen gerecht geworden ist (3.).

\section{Das, geistige Eigentum': Autorschafts- und Werkbegriffe, die Netzliteratur und neue ästhetische und juridische Standards}

Es dauerte etwa drei Jahrhunderte, bis sich nach der Etablierung der Druckerpresse mit beweglichen Lettern ein differenzierter Literaturmarkt etablierte. Gegen die Praxen der 1770er Jahre, die Reinhard Wittmann als „Nachdruckzeitalter“"4 bezeichnet, wurde die Vorstellung des, freien Schriftstellers' gesetzt, der ein ,geistiges Eigentum' an seinen Texten besitze. Im Hintergrund dieser Konstruktion eines, geistigen Eigentums' stehen die Subjektphilosophie von Denkern wie Immanuel Kant und Johann Gottlieb Fichte und die genieästhetische Vorstellung, ein ,Autor ${ }^{6}$ schöpfe aus sich selbst heraus ein ,Werk'.

Damit wird der Wert individueller Textschöpfung besonders hoch angesetzt und eine Herrschaft des Autors über sein Werk begründet: Autorschaft wird Werkherrschaft. ${ }^{5}$ Diese Konzentration auf den Schutz des Urhebers aus der spezifischen Vorstellung kultureller Produktion wirkt bis heute fort im deutschen Urheberrecht: „Werke im Sinne dieses Gesetzes

3 Vgl. dazu u.a. Ernst, Autor-Leser-Texte, in: Böck et al., Lesen X.0, 145; Ernst, Kritik des Werkbegriffs, in: Jannidis, Digitale Literaturwissenschaft. Das folgende Kapitel ist eine stark komprimierte Zusammenfassung von Teilen dieser Aufsätze.

4 Wittmann, Geschichte des deutschen Buchhandels, $131 \mathrm{f}$.

5 Vgl. Bosse, Autorschaft ist Werkherrschaft. 
sind nur persönliche geistige Schöpfungen." ${ }^{6}$ Im literarästhetischen Diskurs werden solche Werke primär als abgeschlossenes Buch gedacht, das als materieller Gegenstand zugleich auch literaturtheoretisch dieses juridische und buchökonomische Geschäftsmodell garantiert.

Schon in der Moderne wurden diese Modelle starker Autorschafts- und Werkbegriffe jedoch ästhetisch (u.a. historische Avantgarde, Kollaborationen, Intertextualität) sowie literaturtheoretisch (u.a. durch Michel Foucault, Roland Barthes oder die Rezeptionsästhetik) problematisiert. Nun etablieren die Potentiale der digitalen Kopie und insbesondere die kommunikative Vernetzung auf den Plattformen der Sozialen Medien im World Wide Web neue Medienverhältnisse, die eine große Herausforderung für die etablierten journalistischen, literarischen und künstlerischen Wertschöpfungsketten darstellen.

Dieser Medienwandel wird einerseits als eine neue „Kultur der Digitalität“ begrüßt und andererseits als ein Medienzeitalter der „Hypnose“ diffamiert; verpönte Kulturtechniken wie die ,Kopie‘ werden unter dem Begriff des „Mashup“ gegen die ,Originalität" in Stellung gebracht: eine Umwertung der Begriffe und bestehender Konzepte. ${ }^{7}$ Jenseits dieser großen kulturtheoretischen Debatten und ihrer Schlagworte zwischen Digitaleuphorie und der Verteidigung des Druckzeitalters ist es fundamental, differenzierte Analysen konkreter Phänomene vorzulegen. Insbesondere sollten die Folgen des digitalen Wandels für Begriffe der Autorschaft, des Werkcharakters und des, geistigen Eigentums' auf unterschiedlichen ästhetischen Feldern wie Film, Fernsehen, Radio oder Musik untersucht werden. Im Bereich der digitalen Literatur rückt dabei insbesondere die ,Netzliteratur in den Fokus.

Simone Winko grenzt die Netzliteratur dezidiert von einer, digitalisierten (Buch-)Literatur ${ }^{\varsigma}$ und einer, digitalen Literatur' ${ }^{\natural}$ b, die beide auch ohne Online-Kommunikation funktionieren könnten. Eine Netzliteratur jedoch ist auf die vernetzte Kommunikation im World Wide Web angewiesen und könne daher "neue Möglichkeiten der Interaktion zwischen Text und Leser, aber auch der Kommunikation von Lesern miteinander" hervorbringen. ${ }^{8}$ Damit vollzieht sich im literarischen Kommunikationsmodell ein fundamentaler Wandel: von einer individuellen Buchautorschaft zur Autor-Leser-Interaktion in Sozialen Medien und von der Zentrierung des

$6 \$ 2$ Abs. 2 S. 1 UrhG.

7 Vgl. Stalder, Kultur der Digitalität; Reuß, Ende der Hypnose; von Geblen, Mashup.

8 Winko, Digitale Literatur, in: Arnold/Beilein, Literaturbetrieb, 292, 294. 
Werks als dem genuinen (Verkaufs-)Gegenstand des Buchmarkts hin zu literarischen Kommunikationsnetzwerken auf Webplattformen.

Als Netzliteratur können sehr unterschiedliche Formen bezeichnet werden. Dazu zählen literarische Weblogs, also Litblogs; die netzliterarische Leser-Leser-Kommunikation in den Bereichen der Fan-Fiction und der Social Reading-Plattformen, die eine Form der Leser-Online-Literaturkritik darstellt. Schließlich können auch Kürzestnarrationen in interaktiven Netzwerken als Netzliteratur gelesen werden: Twitteratur oder Instapoesie. Für diese vernetzte Produktion, Distribution und Modifikation von kulturellen Werken erweisen sich jedoch die historisch gewachsenen Vorstellungen des ,abgeschlossenen Werks', der ,genialischen Autorschaft' und des ,geistigen Eigentums' eher als hinderlich denn als produktiv.

Yochai Benkler hat in The Wealth of Networks schon 2006 die Spannung zwischen den bestehenden (urheber-)rechtlichen Regelungen einerseits und den Erfordernissen einer digitalen Kultur andererseits beschrieben. ${ }^{9}$ Tatsächlich benötigen die Netzliteratur und generell die Formen der vernetzten kulturellen Produktion andere rechtliche Standards. Erstens sollten sie im Idealfall im Sinne des Open Access frei verfügbar, nachnutzbar und modifizierbar sein (beispielsweise unter einer Creative Commons-Lizenz wie CC BY), ${ }^{10}$ um überhaupt eine breite netzliterarische bzw. kulturelle Kommunikation zu ermöglichen. Zweitens wäre im Sinne einer Kommunikation, die dem offenen Verhältnis von Autoren bzw. Kulturproduzenten und Lesern angemessen wäre und dem Ideal eines souveränen Datenmanagements entspräche, eine weitgehende Offenlegung der erhobenen Lesedaten und ibrer Nutzung geboten. Hierzu gehört zudem die offenere Gestaltung von Schnittstellen und Zugriffsmöglichkeiten sowie die Transparenz von Algorithmen.

Auch im rechtswissenschaftlichen Diskurs werden die „Unzulänglichkeiten des urheberzentrierten Individualitätskonzepts"11 zunehmend hervorgehoben. Malek Barudi zeigt in Autor und Werk, dass die vom Urheberrecht zentral gesetzte ,Prägetheorie', der zufolge sich in einem literarischen Werk die individuelle Autorschaft erweise, nicht angemessen überprüfbar sei. Anstelle dessen plädiert er für den Bezug auf das Werk selbst und seine Differenz von anderen Werken. ${ }^{12}$

9 Benkler, The Wealth of Networks, $469 \mathrm{f}$.

10 Vgl. Creative Commons, Creative Commons, https:/creativecommons.org/ (zuletzt abgerufen am 30.5.2020).

11 Barudi, Autor und Werk, 199.

12 Vgl. ebd., 200. 
Für den spezifischen Bereich der Netzliteratur sind auch die Arbeiten von Axel Metzger relevant, der das Urheberrecht und die Freie oder Open Source Software (FOSS) miteinander in ein Verhältnis setzt. Eine Vergleichbarkeit der Phänomene ist gegeben, da beide auf die kollaborative Produktion sowie die offene Verfüg- und Nachnutzbarkeit angelegt sind. Schon 2011 konstatierte Metzger eine starke Differenz zwischen „dem im Gesetz mit Nachdruck betonten Schöpferprinzip und der weniger prinzipientreuen Rechtsrealität" ${ }^{13}$ insbesondere für ",dezentrale Urheberkollekti$\mathrm{ve}^{\text {"14 }}$ sei das Urheberrecht wenig hilfreich. In einem späteren Beitrag untersucht er diese Frage genauer am Beispiel der Wikimedia-Foundation und stellt fest, dass die (offene) Lizenzierung von Inhalten die führende Tendenz sei. Allerdings fordert er zugleich ein, dass insbesondere „die verschiedenen Funktionen und Vorrechte, die Akteure [...] wahrnehmen“, genauer untersucht werden müssten, auch fehle es ,an einer umfassenden Kartographie der internen Community-Regularien" sowie einer Analyse der „Auswirkungen dieser Regularien auf die kreativen Prozesse“. ${ }^{15}$

Es ist eine transdisziplinäre Aufgabe, diese Multiplikation von Autorschaftsrollen und die Differenzierung kollaborativer Produktionsprozesse zu analysieren. Die Literaturwissenschaft kann hierzu einen wichtigen Beitrag leisten. Nun könnte man sich an dieser Stelle auf die Fan-Fiction als einer wuchernden Produktionsform auf Leser-Leser-Plattformen oder die Twitteratur als Beispiel der literarischen Produktion in einem großen Sozialen Medium konzentrieren, dazu gibt es jedoch bereits andere Studien. ${ }^{16}$ Dieser Beitrag wird sich exemplarisch einem Buchprojekt widmen, das sich als Crowdfunding- und Crowdsourcing-Projekt verstanden hat und in besonderer Weise und als Erstes netzliterarische Modelle der Produktion, Distribution, Lizenzierung und Buchökonomie ausprobiert hat. Damit eignet es sich in besonderer Weise als Analysegegenstand, an dessen Beispiel man nach einem Tipping Point für die Entwicklung netzliterarischer Geschäfts- und Rechtsmodelle fragen kann.

13 Metzger, Erosion des Schöpferprinzips, in: Leible/Ohly/Zech, Wissen - Märkte Geistiges Eigentum, 79, 82.

14 Ebd., 92.

15 Metzger, Open Source, in: Grünberger/Leible, Urheberrecht und Nutzerverhalten, $115,127$.

16 Vgl. Summerer, ,Illegale Fans'; Reißmann/Klass/Hoffmann POP. Kultur \& Kritik 2017, 156. 
2. Crowdfunding und Crowdsourcing des Buchprojekts Eine neue Version ist verfügbar

Im Anschluss an internationale Crowdfunding-Plattformen wie Kickstarter oder Unbound, ${ }^{17}$ das mit dem Slogan "Authors pitch their ideas. You choose which books get written." antrat, haben sich auch in Deutschland Plattformen etabliert, die erfolgreich Buchprojekte unterstützen konnten. Über Startnext - Crowdfunding für Projekte und Startups ${ }^{18}$ sind bereits mehrere Buchprojekte erfolgreich gefördert worden, u.a. der Facebook-Roman Zwirbler (mit $15.380 €$ von 144 Unterstützern) und die Sachbücher Das neue Spiel - Nach dem Kontrollverlust (20.467 €/668 Unterstützer), Drachenväter. Die Geschichte des Rollenspiels (20.511 €/428 Unterstützer) sowie - als erstes Projekt mit einem solch großen Erfolg - Eine neue Version ist verfügbar (14.182 €/350 Unterstützer). ${ }^{19}$

Im Folgenden soll exemplarisch das Projekt Eine neue Version ist verfügbar (ENVIV) von Dirk von Gehlen analysiert werden, das zwar - im Gegensatz beispielsweise zu Zwirbler - kein literarisches Werk, sondern ein Sachbuch ist, allerdings dezidiert den Anspruch formuliert, ,in einem Experiment gemeinsam mit den Lesern die Idee von Kultur als Software auszuarbeiten. Wie funktioniert ein Buch, das in Versionen entsteht und diese Versionen dokumentiert und diskutiert?" ${ }^{20}$ Damit formuliert das Projekt selbst den Anspruch, in vierfacher Weise als Tipping Point zu fungieren: Erstens, indem es die frühere Subskription literarischer Werke als Crowdfunding in den digitalen Raum überführen möchte; zweitens, indem es dieses Crowdfunding mit dem über die bloße Subskription hinausgehenden Anspruch verknüpft, die Förderer auch am Schreibprozess teilhaben zu lassen (Crowdsourcing); drittens, indem das Werk inhaltlich diesen Wandel als eine neue Form kultureller Produktivität reflektiert; und schließlich viertens, indem es seinem Titel gemäß für seine eigene Fort- und Umschreibung nachnutzbar sein möchte.

17 Kickstarter, https://www.kickstarter.com/; Unbound, https://unbound.com (beide zuletzt abgerufen am 30.5.2020).

18 Startnext, https://www.startnext.com (zuletzt abgerufen am 30.5.2020).

19 Vgl. Hillenbrand/Lischka, Drachenväter, https://www.startnext.com/drachenvaeter; Seemann, Das neue Spiel, https://www.startnext.com/ctrlverlust/; Teglasy, Zwirbler, https://www.startnext.com/zwirbler; von Geblen/Drey/ßig, Eine neue Version ist verfügbar, https://www.startnext.com/neueversion, folgend nur „ENVIV (Startnext)“ (alle zuletzt abgerufen am 30.5.2020).

20 Von Gehlen/Dreyßig, ENVIV (Startnext); Hervorh. im Orig. (allerdings von Fettdruck auf Kursivierung umgestellt). 
Dirk von Gehlen arbeitet als Journalist bei der Süddeutschen Zeitung und hat 2011 mit Mashup. Lob der Kopie beim Suhrkamp Verlag ein Sachbuch vorgelegt. Sein Plädoyer für einen neuen Begriff des Originals und dafür, dass die digitale Gesellschaft „ihren Mitgliedern die Möglichkeiten der Teilhabe und Partizipation (...) und (...) auf das Kopieren " einräumen müsse, denn „[w]ir können nicht nicht kopieren“, 21 ist in vielen Medien besprochen worden. Insbesondere der Widerspruch, somit ein Plädoyer gegen traditionelle Rechtsmodelle der Buchkultur urheberrechtlich geschützt bei einem der wichtigsten deutschen Verlagshäuser veröffentlicht zu haben, habe sein Nachfolgeprojekt prädestiniert für ein anderes Vorgehen. Dass er die ,Verflüssigung bzw. Versionierung von Texten' nicht nur inhaltlich reflektieren, sondern auch buchökonomisch und rechtlich umsetzen möchte, habe ihn zum Crowdfunding gebracht: „Ich habe festgestellt, dass ich das nicht in einem klassischen Verlag veröffentlichen kann, sondern als Projekt auf eigene Faust machen muss. So bin ich zum Crowdfunding gekommen."22 Zunächst soll im Folgenden dargestellt werden, welche Ansprüche das Projekt an sich selbst formuliert, um anschließend zu überprüfen, welche Ansprüche es tatsächlich eingelöst hat.

Am 22. Oktober 2012 startet der zweimonatige Finanzierungszeitraum für das Crowdfunding-Projekt Eine neue Version ist verfügbar auf der Plattform Startnext, als Fundingziel werden $5.000 €$ avisiert, das Buch soll bei einer erfolgreichen Förderung schon im Mai 2013 vorliegen. Inhaltlich solle es „, $[\mathrm{u}] \mathrm{m}$ eine neue Art, Bücher zu schreiben“, gehen, Kunst und Kultur sollten in der digitalen Welt „[a]ls Prozess, nicht als Produkt“ verstanden werden, und genau dies solle das Buchprojekt „mit Ihrer Hilfe in die Tat umsetzen." ${ }^{23}$ In einem zweiminütigen Pitchvideo von Tim Klimeš nobilitiert sich der Autor zunächst, indem er auf seine Arbeit bei der Süddeutschen Zeitung und seine Buchveröffentlichung beim Suhrkamp Verlag verweist. Dann präsentiert er seine Projektidee und schließlich acht namhafte Unterstützer des Projekts, die allesamt selbst im Kontext oder über Fragen des digitalen Medienwandels arbeiten. Dazu gehören unter anderem die Professoren Urs Gasser (Harvard), Felix Stalder (Zürich) und Caja Thimm (Bonn) oder die Autoren Peter Glaser und Kathrin Passig (Berlin), die fast alle sichtbar das Mashup-Buch in ihren Händen halten. Auf diese

21 Von Geblen, Mashup, 175, 179.

22 Von Gehlen, zit. n. Brückner, Digitalisierung von Kunst und Kultur, 53.

23 Von Gehlen/Dreyßig, ENVIV (Startnext). 
Weise verleihen sie mit den Insignien der ,Gutenberg-Galaxis‘ dem Digitalprojekt symbolisches Kapital. ${ }^{24}$

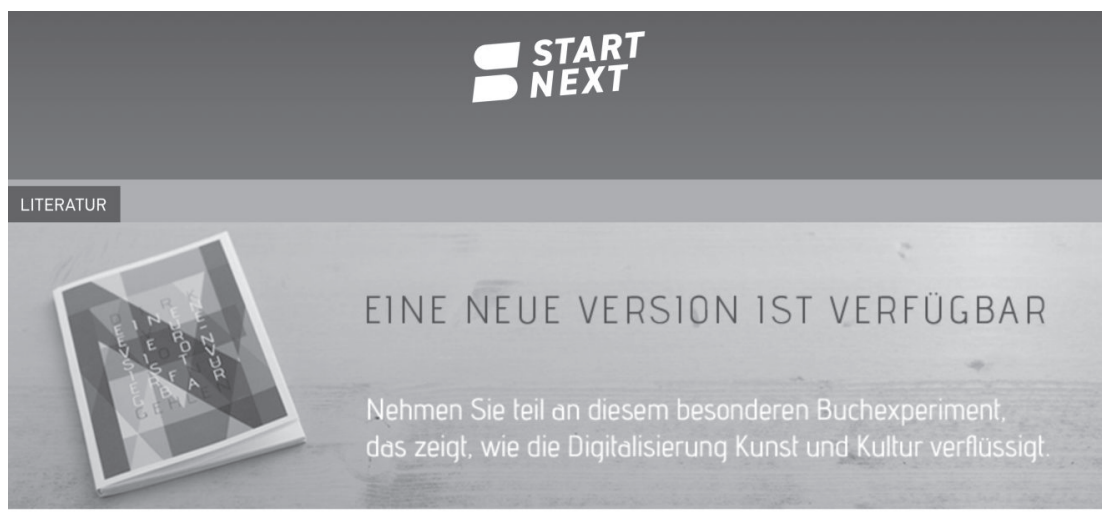

Das neue Buchprojekt des SZ-Redakteurs und Suhrkamp-Autors Dirk von Gehlen sucht Antworten auf die Frage: Wie verändert die Digitialisierung Kunst und Kultur? Meine These: Sie taut sie auf. Sie verflüssigt sie! "Eine neue Version ist verfügbar" will beschreiben und selber zeigen, wie das die Denk- und Geschäftsmodelle von Filmen, Musik und Büchern verändern kann. Helfen Sie mit, eine neue Version verfügbar zu

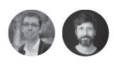

Neue Version machen!

$\begin{array}{lll}14.182 € & 350 & \begin{array}{l}\text { Projekt } \\ \text { erfolgreich }\end{array} \\ 5.000 € \text { Fundingziel } & \text { Unterstützer*innen }\end{array}$

Das Projekt Eine neue Version ist verfügbar auf Startnext. ${ }^{25}$

Für die Unterstützer sind zwölf verschiedene ,Dankeschöns' vorgesehen, die von einer Basis-Unterstützung für $5 €$ (namentliche Nennung im Buch und Erhalt eines ENVIV-Bildschirmhintergrunds) bis zu einer SponsoringUnterstützung in Höhe von $2.500 €$ (die tatsächlich einmal erworben wird) reichen. Die meisten Unterstützer entscheiden sich für die Pakete ,Basis-eBook' (12 €/85 Personen), ,Basis-Buch' (20€/98) und ,PremiumBuch' (30€/135) - wobei bemerkenswert ist, dass sich die Mehrheit der Unterstützer bei diesem Digitalprojekt für ein gedrucktes Buch als ,Dankeschön' entscheiden und darunter wiederum eine Mehrheit für eine um

24 Vgl. von Geblen/Klimeš, ENVIV (Video).

25 Von Gehlen/Dreyßig, ENVIV (Startnext), Auszug. 
$50 \%$ teurere Variante mit einem individuellen Cover (das ist die einzige Differenz zwischen Basis- und Premium-Buch). Ein großer Teil der an innovativen digitalen Buchprojekten Interessierten bleibt also eigentlich bibliophil in einem ganz haptischen Sinne. Das Finanzierungssetting erweist sich jedenfalls als sehr erfolgreich, denn letztendlich haben 350 Unterstützer das Projekt erfolgreich mit insgesamt 14.182 € gefördert, das Crowdfunding dieses Buchprojekts ist somit gelungen. ${ }^{26}$

Wie aber gestaltet sich das fertige Buchprojekt und wie verlief das angekündigte Crowdsourcing? Die erstveröffentlichte Textversion konstatiert, dass sie vor dem Hintergrund des digitalen Medienwandels zeigen wolle, „wie das Ablösen der Daten von ihrem Träger auch ihre Form verändert. Sie tauen dadurch auf, verflüssigen sich. [...] Ein Film, ein Song, ein Text (und alle digitalisierten Werkstücke) können ihren besonderen Zauber künftig immer mehr aus dem Prozess ihres Entstehens ziehen denn einzig aus dessen Resultat. Diesem Prozess der Verflüssigung widmet sich dieses Buch. “27 Strukturell lässt sich das Buch zum Thema ,digitale Verflüssigung der Kultur' als ein Hybrid aus der durchgängigen Argumentation eines Autors einerseits sowie von verschiedenen Gesprächen des Autors mit Expertinnen und Experten zum Thema andererseits bezeichnen. Diese Mischung aus linearer Argumentation und vielstimmiger Reflexion ermöglicht, sowohl die Kernthese des Buches theoretisch zu reflektieren als auch anhand verschiedener Beispiele zu untermauern.

Daneben treten traditionelle Buchelemente wie ein Glossar und ein Vorwort, jedoch auch ungewöhnliche Elemente: ein Post Scriptum: Ein Blick zwischen die Zeilen, das die Einwände des mit dem Erlös des Crowdfundings bezahlten externen Lektors gegen einzelne Thesen und Verfahrensweisen des Buches offenlegt, sowie eine Übersicht über die Unterstützer. ${ }^{28}$ Diese beiden Kapitel können als direkter Einfluss des Crowdfundings auf die Buchinhalte bewertet werden: Durch die Offenlegung der Lektoreneinwände werden Schwächen und die Konstruiertheit des Textes transparent gemacht, was wiederum zur kritischen Diskussion und Fort-

26 Der Verfasser dieses Aufsatzes hat die ,Basis-Buch'-Variante für $20 €$ erworben, um als Teil des 350-köpfigen Unterstützerkreises das Projekt und insbesondere seine Produktionsphase im Sinne einer teilnehmenden Beobachtung begleiten zu können. Zudem sollte darauf hingewiesen werden, dass der Verfasser bereits seit langer Zeit mit Dirk von Gehlen bekannt ist. Die folgenden kritischen Ausführungen mögen jedoch zeigen, dass der Verfasser trotz dieser Nähe eine angemessene analytische Distanz eingenommen hat.

27 Von Gehlen, ENVIV (Buch), 9 und $15 \mathrm{f}$.

28 Vgl.ebd. 191-217.

https://doi.org/10.5771/9783748910664-81, am 26.04.2023, 16:44:45 
schreibung des Textes einlädt; durch die 356-köpfige Namensliste und die achtzig Fotoportraits der Unterstützerinnen und Unterstützer wird transparent gemacht, wer das Buchprojekt überhaupt materiell ermöglicht hat.

Inwiefern wurden diese Unterstützer und die 25 namentlich genannten besonders wichtigen Projektmitarbeiter oder -unterstützer ${ }^{29}$ jedoch tatsächlich in einen kollaborativen Produktionsprozess eingebunden? Letztlich wurden die digitalen Möglichkeiten zur kollaborativen Produktion des Buches nur sehr eingeschränkt genutzt, das Buch ist eher als das Werk eines singulären Autors denn als das Ergebnis eines kollaborativen Schreibprozesses zu bewerten. Zwar informierte der Autor seine Unterstützer regelmäßig per Email über den Stand seines Schreibprozesses, er stellte PDFs der gerade entstandenen Kapitel und Audiodateien der Lektoratsgespräche zur Verfügung, auf die seine Unterstützer per Email mit Anregungen und Hinweisen reagieren konnten. Nur einmal fand ein Live-Schreib-Experiment statt, das allerdings eher eine Diskussion über die Textproduktion als über den entstehenden Text selbst war. ${ }^{30}$ Somit gab es keinen kontinuierlichen gemeinsamen Schreibprozess und auch die Nutzung der per Email eingereichten Anregungen durch den Autor blieb intransparent (in einem Interview erklärt von Gehlen allerdings, dass sich bis zu zehn Prozent der Unterstützer „mit Hinweisen und Kommentaren“ beteiligt hätten). ${ }^{31}$

29 Vgl. ebd., 223.

30 Vgl. von Gehlen, ENVIV (Live-Schreib-Experiment).

31 Vgl. Brückner, Digitalisierung von Kunst und Kultur, 53. Am 21.12.2012 wurden die Unterstützer über die erfolgreiche Finanzierung des Projekts informiert. Der Autor schickte dann die jeweils entstandenen (Erstversionen der) Kapitel und Interviews sowie Audiodateien der Lektoratsgespräche am 8.1., 20.1., 28.1., 3.2., 6.2., 17.2., 7.3. und 22.3.2013 an die Unterstützer. Am 26.3. erhielten sie erstmals das gesamte Buchprojekt, am 6.4. und 21.4. Informationen zum finalen Lektorat und am 23.4. und 1.5.2013 Nachrichten zum Stand der Druckfahnen und zur Finaldatei. Der Launch des Buchprojekts fand am 10.-12.5.2013 bei einer Konferenz zum Buchprojekt in der Evangelischen Akademie Tutzing statt, auf der Unterstützer wie u.a. Udo Hahn, Stefan Plöchinger, Felix Stalder, Leander Wattig und Nicola Wessinghage das Projekt mit dem Autor reflektierten und diskutierten. 


\title{
ERMÖGLICHT DURCH DIE UNTERSTÜTZER DES PROJEKTS „EINE NEUE VERSION IST VERFÜGBAR" AUF STARTNEXT.DE/NEUEVERSION IM WINTER 2012
}

\author{
exklusive Erstauflage
}

München, 2013

\section{Schriften: Adobe Garamond Pro, Advent}

Papier: Target

\section{Druck: DMHM Druckerei \& Medienhaus Maier \\ Lektorat: Bernd Klöckener}

Gestaltung und Satz: SQUIECH Design

,Verlagsangabe der gedruckten Unterstützerversion von

Eine neue Version ist verfügbar. ${ }^{32}$

Die Besonderheit des Projekts wäre somit nicht ein kollaborativer Schreibprozess, sondern vielmehr, dass sich ein starkes Autorsubjekt zugleich die Verlagsfunktionen angeeignet hat, da die traditionellen Buchverlage sich weigerten, produktiv mit den Potenzialen des digitalen Publizierens umzugehen. Die zusätzliche Übernahme bzw. Organisation von Verlagsaufgaben (Lektorat, Gestaltung, Satz, Druck) durch den Autor, der somit als hauptberuflicher Journalist auch noch Buchautor und Kleinverleger in Personalunion war, wurde von ihm allerdings als sehr belastend beschrieben. Bereits im Live-Schreib-Experiment gesteht von Gehlen: „Ich will glaube ich - kein Buch mehr ganz allein schreiben. Also ohne die Unterstützung eines Verlags in den ganzen organisatorischen Fragen. “33 Diese Selbsterkenntnis ist zwar nachvollziehbar, widerspricht allerdings den Ausgangsprämissen des Projekts, das gerade Schreib- und Veröffentlichungsprozesse jenseits traditioneller Verlagsstrukturen experimentell erproben wollte. Konsequent ist es allerdings, dass das Buch unter der Creative

33 Von Gehlen/Unterstützer, ENVIV (Live-Schreib-Experiment; unv., erh. b. Verf.), 7. 
Commons-Lizenz BY-NC-SA veröffentlicht wird, die es ermöglicht, das Buch zu verbreiten und zu bearbeiten, sogar zu neuen Versionen. Dabei muss allerdings der Name des Autors genannt, die Nichtkommerzialität eingehalten und die Weitergabe unter gleichen Bedingungen beachtet werden.

Eine massenhafte Produktion neuer Versionen aus dem Buch Eine neue Version ist verfügbar hat es jedoch nicht gegeben, wenngleich das Buch in Fernsehen, Radio, Zeitungen und digitalen Medien intensiv besprochen wurde. Überraschenderweise hat der Autor dem Metrolit Verlag erlaubt, im September 2013 ein Update des Buches als gedruckte Version und als EBook (für 12,99€ bzw. 7,99 €) zu veröffentlichen, in der u.a. das kritische Lektoratskapitel fehlt. Auf diese Weise ist das gelungene Crowdfundingund das problematische Crowdsourcing-Projekt Eine neue Version ist verfügbar allerdings wieder von den traditionellen Verlagsstrukturen absorbiert worden und der Autor erhielt einen üblichen Verlagsvertrag.

\section{Nach dem geistigen Eigentum: Geschäfts- und Rechtsmodelle der Netzliteratur}

Die Konstruktion des, geistigen Eigentums' ist historisch auf spezifische ästhetische und philosophische Vorstellungen ,starker Autorschaft', ,innerlicher Produktivität ${ }^{\varsigma}$ und ,abgeschlossener Werke' aufgebaut. Diese Vorstellungen sind künstlerisch und literaturtheoretisch bereits in vielfältiger Weise in Frage gestellt worden, der digitale Medienwandel und seine neuen Kopier- und Kommunikationspotenziale delegitimiert diese Vorstellungen zusätzlich.

Im Bereich der Literatur etablieren sich gegenwärtig mit der Netzliteratur literarische Formen, die die Kommunikationsmöglichkeiten der Sozialen Medien produktiv nutzen. Klare Unterscheidungen wie ,Autor' vs. ,Leser'sowie die Vorstellung , abgeschlossener Werke' lassen sich medientheoretisch nicht mehr in der Form halten, wie sie noch in der Gutenberg-Galaxis für die Produktion, Distribution und Rezeption gedruckter Bücher fundamental waren. Dabei zeigt sich, dass das bestehende Urheberrecht die Praxen der Netzliteratur in problematischer Weise behindert (z.B. die Fortschreibung bestehender Werke in der Fan-Fiction). Im Gegensatz dazu wäre es wichtig, andere rechtliche und kulturelle Standards für die netzliterarische Produktivität zu etablieren, die die Leser-Autoren als Nutzer Sozialer Medien gegenüber den Plattformanbietern emanzipieren (wie auch die Einführung des , geistigen Eigentums' historisch eine Emanzipation der Autoren ermöglichte). 
Als netzliterarisches Fallbeispiel für diesen Umschlag in eine Medienepoche nach dem geistigen Eigentum haben wir ein Buchprojekt untersucht, das auf gleich vier Ebenen diesen Tipping Point markieren möchte. Tatsächlich hat das Crowdfunding- und Crowdsourcing-Projekt Eine neue Version ist verfügbar von Dirk von Gehlen 2012 und 2013 in herausragender Weise gezeigt, dass buchökonomisch die Subskription von Buchprojekten auch auf digitalen Plattformen erfolgreich durchgeführt werden kann. Die drei weiteren selbst formulierten Ansprüche des Projekts konnten jedoch nur in Teilen oder in einer inkonsistenten Weise eingelöst werden.

Produktionsästhetisch ist das geplante Crowdsourcing nur teilweise umgesetzt worden: Zwar konnten die 350 Unterstützer den Produktionsprozess aus der Nähe verfolgen, ein echter Mitschreibeprozess hat sich jedoch nur bedingt ergeben. Damit reflektiert das Buch zwar inhaltlich die kollaborative und verflüssigte Produktionsweise in einer digitalen Kultur, bezieht aber nur partiell die von den Unterstützern in diesem Sinne gemachten Beiträge ein (allerdings gibt es ein Kapitel, das die Einwände des ins Projekt eingebundenen Lektors transparent macht). Schließlich ist auch die rechtliche Form des Buches ambivalent: Zwar steht die am Projektende finalisierte Buchversion unter der Creative Commons-Lizenz CC BY-NC-SA, jedoch gab es erstens keine Vielfalt neuer Versionen von Eine neue Version ist verfügbar, zweitens hat der Autor seine Tätigkeit als Selbstverleger im Nachhinein problematisiert und das Buch auch in einem traditionellen Sinne beim Metrolit Verlag lizenziert (und seine folgenden Buchprojekte wieder mit Verlagen wie Matthes \& Seitz und Piper realisiert).

Aus diesen Analyseergebnissen lässt sich konkludieren, dass im Bereich der Literatur dieser literaturbetriebliche Tipping Point um 2012/13 zwar einerseits zeigt, welche buchökonomischen Potenziale die digitale Netzwerkkommunikation hervorbringt, wie verhaftet Autoren und Leser jedoch produktionsästhetisch, inhaltlich und rechtlich noch immer dem Literaturmarkt der gedruckten Bücher sind. Dennoch hat dieses Projekt gezeigt, dass auch renommierte Autoren heute nicht mehr auf Verlage angewiesen sind, um Buchprojekte erfolgreich zu finanzieren und zu realisieren. Die Plattform Startnext ist inzwischen etabliert und wird beispielsweise von Verlagen wie Hanser genutzt, um hybride Formate zu produzieren. ${ }^{34}$

Somit zeigt das Fallbeispiel Eine neue Version ist verfügbar, dass neben dem traditionellen Buchmarkt und seinem Wertschöpfungsmodell auch andere Modelle der Buchproduktion, -distribution und -rezeption möglich

34 Vgl. Ernst, Autor-Leser-Texte, in: Böck et al., Lesen X.0, 160-163. 
sind, die von veränderten Werkbegriffen und einer fundamental anderen Autor-Leser-Kommunikation ausgehen. Zudem verschiebt sich der Ansatzpunkt für monetäre Erlöse vom fertigen Produkt hin zum kulturellen Produktionsprozess. Es ist wichtig, diese neuen Modelle neben bzw. nach dem geistigen Eigentum durch eine produktive Zusammenarbeit von Rechtsund Literatur- sowie Medienwissenschaft zu begleiten und dabei weitere Fallstudien zu anderen Feldern der Netzliteratur anzufertigen. Nur so können Rechtsdiskurs und kulturelle Praxen in einer ausgewogenen Verbindung gehalten werden.

\section{Literatur}

\section{Primärliteratur}

Hillenbrand, Tom / Lischka, Konrad, Drachenväter. Die Geschichte des Rollenspiels und die Geburt der virtuellen Welt, https://www.startnext.com/drachenvaeter (alle Webseiten zuletzt abgerufen am 30.5.2020).

Kickstarter, https://www.kickstarter.com/.

Seemann, Michael, Das neue Spiel. Nach dem Kontrollverlust, https://www.startnext. $\mathrm{com} / \mathrm{ctrlverlust/.}$

Startnext, Crowdfunding Plattform für Projekte und Startups, https://www.startnext .com.

Teglasy, Gergely, Zwirbler, https://www.startnext.com/zwirbler.

Unbound, Breaking barriers through crowdfunded books, https://unbound.com/.

Von Gehlen, Dirk, Eine neue Version ist verfügbar, München 2013 (= ENVIV (Buch)).

Von Gehlen, Dirk, Eine neue Version ist verfügbar - das neue Buch von Dirk von Gehlen, http://www.enviv.de (= ENVIV (Webseite)).

Von Gehlen, Dirk / Dreyßig, Florian, Eine neue Version ist verfügbar (online seit dem 22.10.2012, https://www.startnext.com/neueversion (= ENVIV (Startnext)).

Von Gehlen, Dirk / Klimeš, Tim, Eine neue Version ist verfügbar. Das Pitch-Video zum neuen Buchprojekt von Dirk von Gehlen (online seit dem 16.10.2012), https://vimeo.com/51523977 (= ENVIV (Video)).

Von Gehlen, Dirk / Unterstützer, Live-Schreib-Experiment (unveröffentlichte PDF, erhältlich beim Autor; = ENVIV (Live-Schreib-Experiment)). 


\section{Sekundärliteratur}

Barudi, Malek, Autor und Werk - eine prägende Beziehung. Die urheberrechtliche Prägetheorie im Spiegel der Literaturwissenschaft, Tübingen 2013.

Benkler, Yochai, The Wealth of Networks. How Social Production Transforms Markets and Freedom, New Haven; London 2006.

Bosse, Heinrich, Autorschaft ist Werkherrschaft. Über die Entstehung des Urheberrechts aus dem Geist der Goethezeit, Paderborn 1981.

Brückner, Claudia, Die Digitalisierung von Kunst und Kultur. Ein Interview mit Dirk von Gehlen zum Thema Self-Publishing, in: Gebhard, Andreas (Hrsg.), Open Everything. Zehn Jahre newthinking. Ein Magazin über digitale Kultur, Netzpolitik und neue Arbeitswelten, Berlin 2013, S. 52-55.

Creative Commons, Creative Commons, https://creativecommons.org/.

Dommann, Monika, Autoren und Apparate. Die Geschichte des Copyrights im Medienwandel, Frankfurt am Main 2014.

Ernst, Thomas, Wem gehören Autor-Leser-Texte? Das geistige Eigentum, netzliterarische Standards, die Twitteraturvon @tiny_tales und das Online-Schreibprojekt morgen-mehr.de von Tilman Rammstedt, in: Böck, Sebastian et al. (Hrsg.): Lesen X.0. Rezeptionsprozesse in der digitalen Gegenwart, Göttingen 2017, S. 145167, https://www.vr-elibrary.de/doi/pdf/10.14220/9783737007450.145.

Ernst, Thomas, Netzliteratur als Soziales Medium. Zur Kritik des Werkbegriffs, in: Jannidis, Fotis (Hrsg.), Digitale Literaturwissenschaft. Proceedings des literaturwissenschaftlichen DFG-Symposiums Digitale Literaturwissenschaft, Villa Vigoni, 9.-13.10.2017, Stuttgart 2020 (im Erscheinen).

Fichte, Johann Gottlieb, Beweis der Unrechtmässigkeit des Büchernachdrucks. Ein Räsonnement und eine Parabel, in: von Fichte, Immanuel Hermann (Hrsg.), Johann Gottlieb Fichtes Sämmtliche Werke. Band VIII: Vermischte Schriften und Aufsätze, Berlin 1846, S. 223-244.

Von Geblen, Dirk, Mashup. Lob der Kopie, Berlin 2011.

Metzger, Axel, Vom Einzelurheber zu Teams und Netzwerken: Erosion des Schöpferprinzips?, in: Leible, Stefan / Ohly, Ansgar / Zech, Herbert (Hrsg.), Wissen Märkte - Geistiges Eigentum, Tübingen 2010, S. 79-92.

Metzger, Axel, Open Source und andere alternative Lizenzmodelle. Nutzung des Urheberrechts als Mittel zur Sicherung freier Kommunikation, in: Grünberger, Michael / Leible, Stefan (Hrsg.), Die Kollision von Urheberrecht und Nutzerverhalten, Tübingen 2014, S. 115-129.

Reißmann, Wolfgang / Klass, Nadine / Hoffmann, Dagmar: Fan Fiction, Urheberrecht und Empirical Legal Studies, POP. Kultur \& Kritik, 6 (2017), S. 156-172.

Reuß, Roland, Ende der Hypnose. Vom Netz und zum Buch, Frankfurt am Main; Basel 2012.

Stalder, Felix, Kultur der Digitalität, 3. Aufl., Berlin 2017.

Summerer, Claudia, ,Illegale Fans‘. Die urheberrechtliche Zulässigkeit von Fan Art,

Berlin; Boston 2015. 
Theisohn, Philipp, Literarisches Eigentum. Zur Ethik geistiger Arbeit im digitalen Zeitalter. Essay, Stuttgart 2012.

Winko, Simone, Am Rande des Literaturbetriebs. Digitale Literatur im Internet, in: Arnold, Heinz-Ludwig / Beilein, Matthias (Hrsg.), Literaturbetrieb in Deutschland, 3. Aufl., München 2009, S. 292-303.

Wittmann, Reinhard, Geschichte des deutschen Buchhandels, 3. Aufl., München 2011.

Woodmansee, Martha, On the Author Effect: Recovering Collectivity, in: Woodmansee, Martha / Jaszi, Peter (Hrsg.), The Construction of Authorship. Textual Appropriation in Law and Literature, Duke 1994, S. 15-28. 